\title{
The influence of cornering on the vortical wake structures of an inverted wing
}

\author{
James Keogh', Graham Doig ${ }^{1,2}$, Sammy Diasinos ${ }^{3}$ and Tracie Barber'
}

\begin{abstract}
The aerodynamic performance of inverted wings on racing-car configurations is most critical when cornering; however, current wind tunnel techniques are generally limited to the straight-line condition. The true cornering condition introduces complexity because of the curvature of the freestream flow. This results in an increase in the tangential velocity with increasing distance from the instantaneous centre of rotation and causes the front wing to be placed at a yaw angle. Numerical simulations were used to consider an $80 \%$ scale front wing when steady-state cornering with radii ranging from $60 \mathrm{~m}$ to $7.5 \mathrm{~m}$, and yaw angles ranging from $1.25^{\circ}$ to $10^{\circ}$. The changes to the pressure distribution near the endplates caused the wake structure to become highly asymmetric. Both the primary longitudinal vortices and the secondary longitudinal vortices differed in strength, and the vortex core positions shifted in the vertical direction and the spanwise direction. The change in the position became more substantial further downstream as the structures tended toward the freestream direction. The effects on the wing surface pressure distribution resulted in the introduction of yawing and rolling moments, as well as a side force and an increase in drag. The results demonstrate the importance of evaluating the cornering condition if that is where a good performance is most sought after.
\end{abstract}

\section{Keywords}

Vehicle aerodynamics, cornering, ground effect, aerodynamics, computational fluid dynamics

\section{Introduction}

\section{Aerodynamic evaluation of bodies while cornering}

In motorsport, aerodynamic devices are used to produce a downforce which increases the tyre adhesion and ultimately enables higher levels of acceleration to be achieved. ${ }^{1,2}$ This permits modern racing cars to corner at much higher speeds.

Despite the fact that the aerodynamic performance is most critical when cornering, designs will typically be evaluated in the straight-line condition, including combinations of yaw. This is largely because the wind tunnel remains the primary tool for aerodynamic development. There have been previous attempts to replicate the cornering condition in a wind tunnel with the use of bent models ${ }^{3}$ and curved test sections, ${ }^{4}$ but these methods are not capable of representing all aspects of true cornering flow. Industry is aware of the limitations of these methodologies. ${ }^{4}$ At present, the true condition has not been achieved experimentally in the public domain, meaning that numerical simulations are typically preferable for this type of analysis.
The real-world conditions experienced by an openwheel racing car have been identified to have a significant effect on the aerodynamic performance. Parameters such as the pitch, the yaw, and the ride height are already known to have dramatic effects. ${ }^{5}$ An entire open-wheel racing-car geometry was numerically analysed for three specific corners at the Fuji Speed Way Circuit. ${ }^{6}$ The study incorporated the changes in the pitch and the ride height, in addition to cornering. Variation occurred in the lift force and the drag force, as well as in the yawing moment and the side force. No

\footnotetext{
'School of Mechanical and Manufacturing Engineering, University of New South Wales Australia, Sydney, New South Wales, Australia

${ }^{2}$ Aerospace Engineering Department, California Polytechnic State University, San Luis Obispo, California, USA

${ }^{3}$ Department of Engineering, Macquarie University, Sydney, New South Wales, Australia
}

Corresponding author:

James Keogh, School of Mechanical and Manufacturing Engineering, UNSW Australia, Sydney, NSW, 2052, Australia.

Email: j.keogh@unsw.edu.au 
further details regarding the flow structures responsible for these changes was presented, and the effects due to cornering were not clearly distinguished from the effects due to other parameters.

Okada et al. ${ }^{7}$ and Tsubokura et al. ${ }^{8}$ demonstrated the importance of evaluating the high-speed cornering condition during the aerodynamic design phase for a commercial vehicle. Considering a medium-sized sedan geometry, the outboard pressure losses were identified that contributed to a negative yawing moment and side force. This caused a damping effect that restrained the vehicle during cornering. For two different geometries the magnitude of this force varied for various vehicle shapes. A temporal variation in the vehicle reacting to the change in the conditions also occurred. A 49\% difference in the aerodynamic damping toward the steering motion existed between the two geometries; this was largely attributed to the increased space around the wheels in the wheel well.

In motorsport, the aerodynamic performance when cornering becomes even more critical. ${ }^{2,9,10}$ Typical racing-car configurations consist of multiple components which interact to produce a desired aerodynamic outcome. The front wing is most likely to have access to relatively clean flow and has a significant influence on the aerodynamic performances of the downstream components.9,11 The vortical wake leaving the front wing then becomes a critical consideration.

\section{Inverted wing aerodynamics}

The most comprehensive set of straight-line experimental results were conducted by Zerihan. ${ }^{12} \mathrm{He}$ used an inverted T026 aerofoil with endplates, considered across various ground clearances. Studies of this geometry were presented by Zerihan, ${ }^{12}$ Zerihan and Zhang, ${ }^{13}$ and Zhang and Zerihan, ${ }^{10,14}$ considering both the single-element configuration and the double-element configuration. Investigations presented the surface pressures, the forces, and the wake measurements, defining several key aerodynamic characteristics.

Close proximity to the ground resulted in increased acceleration of the flow beneath the inverted suction surface, creating a strong low-pressure region beneath the wing surface. Near the midspan location the flow tended toward a two-dimensional state and the adverse pressure gradient increased as the ground clearance was reduced. A critical point was reached where significant trailing-edge separation resulted in the occurrence of the 'downforce loss phenomenon' at approximately $h / c=0.112$.

The flow near the endplate was characterized by the primary and secondary vortices, as well as by number of smaller flow structures. ${ }^{10}$ The primary vortex formed inside the endplate, as shown in Figure 1, owing to the large pressure gradient. The primary vortex was identified as an important flow structure for operating efficiently in close proximity to the ground. The vortex alleviated the adverse pressure gradient and permitted lower ground clearances to be achieved. ${ }^{15}$ The secondary vortex formed outside the upper edge of the endplate. Increased pressure inside the endplate over the pressure surface resulted in a smaller pressure gradient, which produced a weaker vortex.

Soso and Wilson, ${ }^{16}$ however, highlighted the sensitivities of an inverted wing to change in the oncoming flow. When a wing was positioned in the wake of a generic racing car, a significant loss in the downforce was found to occur.

These findings, together with the little research conducted into aerodynamics when cornering, ${ }^{6-8}$ strongly suggest that a significant change will occur for an inverted wing in the cornering condition. In a practical sense, understanding the aerodynamic performance in

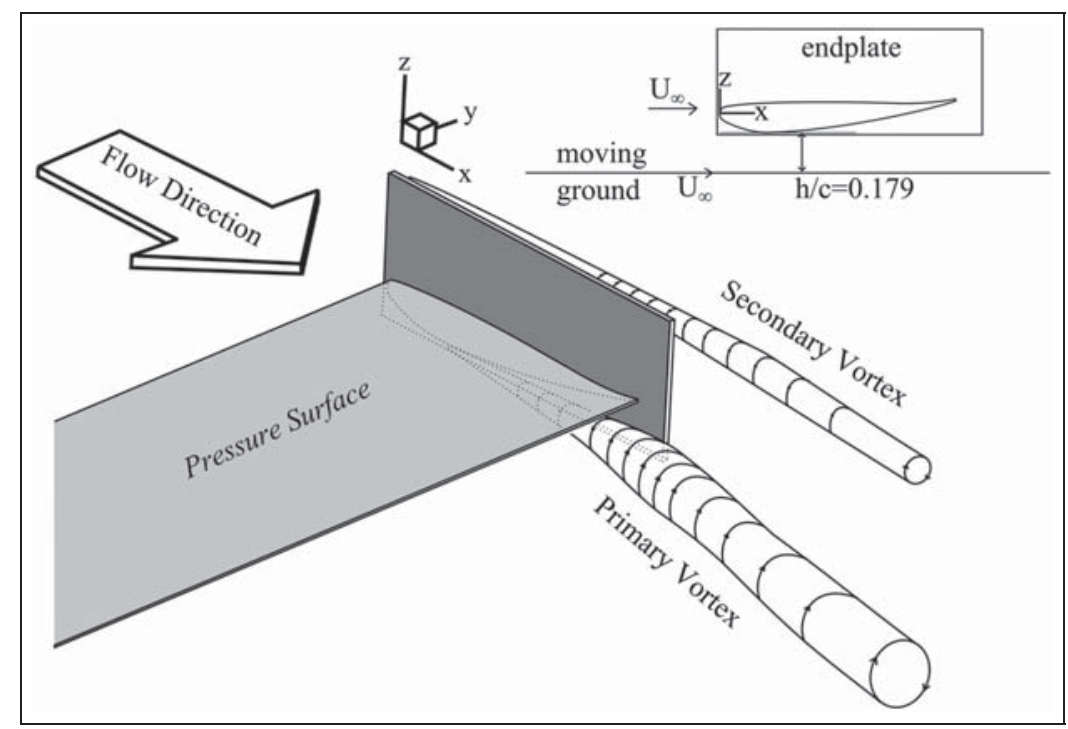

Figure I. Location of the primary vortices and the secondary vortices in the straight-line condition. 


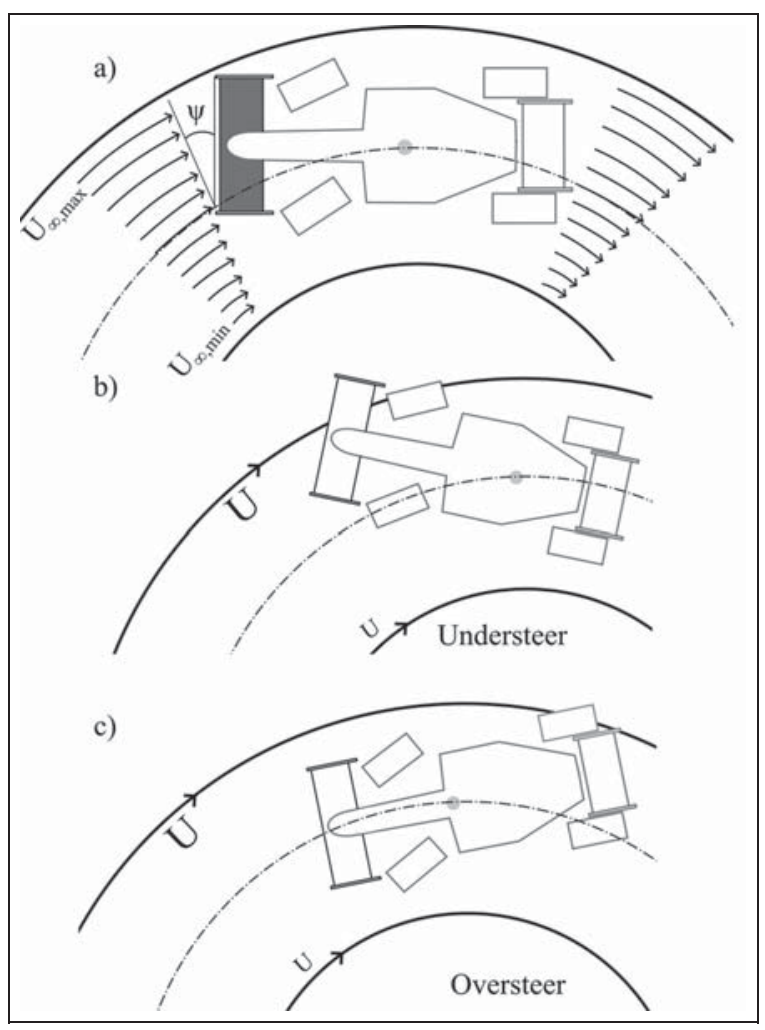

Figure 2. (a) The cornering flow conditions; (b) the effect of understeer toward the flow seen by the front wing; (c) the effect of oversteer toward the flow seen by the front wing.

this condition could be argued as more critical than in the straight-line condition. ${ }^{4}$

\section{Dynamics of cornering}

Modern aerodynamicists have become familiar with a stationary model where the flow field is in motion. When cornering, obviously this relative motion is no longer in a straight line. As the vehicle follows a curved path, so does the flow relative to the vehicle, as is shown in Figure 2(a). The relative velocity of the flow increases with increasing distance from the centre of rotation. In percentage terms, corners with tighter radii will increase the velocity gradient across the span. The flow curvature will also vary and is greater as it becomes closer to the centre of rotation. The variation in the curvature means that the yaw angle of the wing will also vary slightly across the span.

The attitude of the vehicle will have a significant effect. Understeer or oversteer, shown in Figure 2(b) and Figure 2(c) respectively, can cause the wing to be correspondingly closer to or further away from the centre of rotation. This causes changes in both the velocity and the effective angle of the oncoming flow.

In corners with the tightest radii, the aerodynamic forces are reduced in magnitude as the speed of the vehicle is limited by the acceleration able to be sustained. Despite this, some racing cars will spend much time in this condition, making small gains very advantageous. ${ }^{4}$ As a result, the aerodynamic performance in this condition can become crucial.

\section{Method}

\section{Numerical method}

The present study utilizes numerical simulations to investigate the aerodynamics of an isolated inverted wing when cornering at a constant radius and a steady state. All results were generated with the use of the commercial finite-volume solver ANSYS Fluent 14.5, ${ }^{17}$ as is prominent throughout industry. Reynolds-averaged Navier-Stokes simulations were used. Previous studies have proven this technique to be effective for simulating the same geometry in the straight-line condition, ${ }^{15,18-21}$ and it remains the preferred technique within industry, as it is more computationally feasible for development. This study represents the first investigation into the aerodynamic performance of an isolated inverted wing in the cornering condition.

The pressure-based implicit coupled solver was utilized to achieve steady-state simulations. Compressibility effects at the simulated Mach numbers were deemed negligible, in accordance with the conclusions of previous studies. ${ }^{19,21,22}$ Simulations were run using a second-order node-based upwinding discretization scheme across 64 processors. Convergence was deemed to be met when the aerodynamic forces ceased to change by more than $0.02 \%$ over 1000 continued iterations, and a point velocity monitor placed near the centre of the primary vortex also ceased to change by more than $0.02 \%$. For all simulations, the scaled residual errors fell below $8 \times 10^{-5}$.

The coordinates of the aerofoil can be found in the thesis by Zerihan. ${ }^{12}$ A chord length of $223.4 \mathrm{~mm}$ and a span of $1100 \mathrm{~mm}$ gave an aspect ratio of 4.92 . The wing also features a rectangular endplate measuring $250 \mathrm{~mm}$ $\times 100 \mathrm{~mm} \times 4 \mathrm{~mm}$. The wing features a blunt trailing edge $1.5 \mathrm{~mm}$ thick. The wing was described as being at an incidence of $3.45^{\circ}$. Since the wing is symmetric, validation and straight-line cases were run for the semispan with a symmetry plane placed at the midspan location.

The present numerical study was validated against the published experimental results ${ }^{12}$. These experiments were conducted in the Southampton Low-Speed Wind Tunnel, which had test-section dimensions of $2.1 \mathrm{~m}$ $\times 1.7 \mathrm{~m}$ with an octagonal cross-section. The oncoming air was reported at $30 \mathrm{~m} / \mathrm{s}$ within an error of $\pm 0.2 \%$. The freestream turbulence intensity was given as $0.2 \%$. An overhead force balance was utilized for measurement of all the forces. For the numerical validation cases conducted in the current study, a simplified rectangular cross-section was utilized that matched the maximum extents of the wind tunnel. ${ }^{23} \mathrm{~A}$ further simplification of the numerical model was the use of a moving ground plane across the entire width of the test 


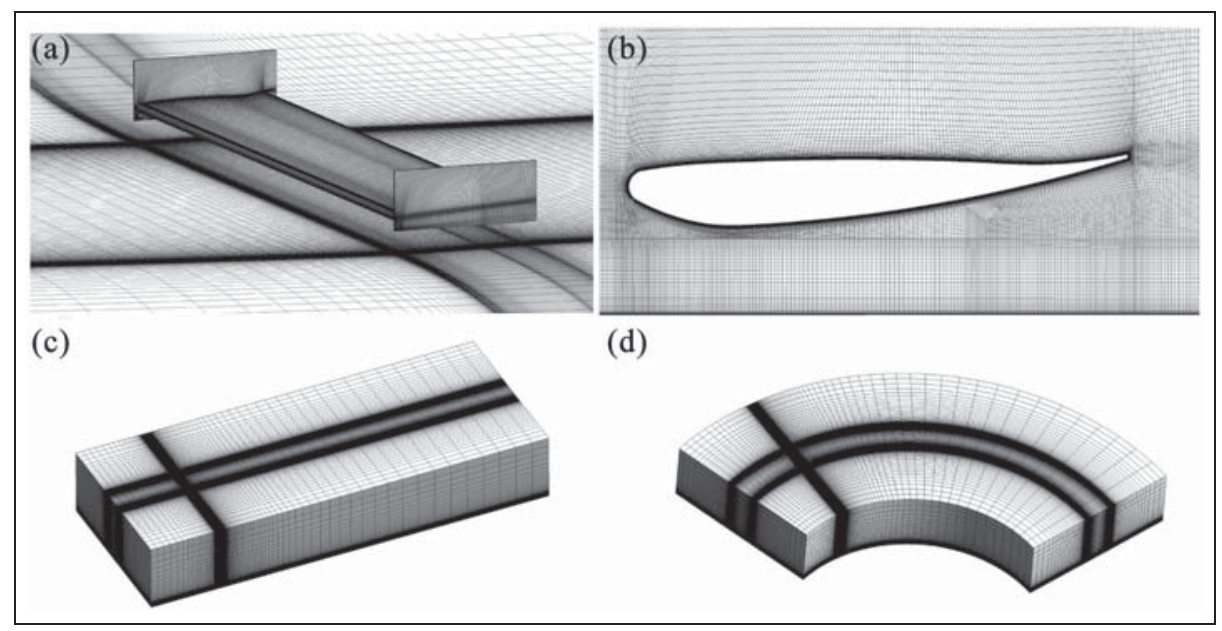

Figure 3. Examples of the mesh structure: (a) isometric view; (b) midspan location; (c) straight-line condition; (d) cornering condition.

section. The boundary layer growth on the walls was not reported from the experiments but can be expected to have a minimal influence owing to the low blockage ratio. As a result, the walls and the roof of the domain were modelled as zero-shear slip walls. The domain was modelled $7 c$ upstream and $15 c$ downstream.

A density of $1.22 \mathrm{~kg} / \mathrm{m}^{3}$ gave a Reynolds number $R e$ of $4.54 \times 10^{5}$ which fell within the reported range for the experimental data. In the published experiments a grit strip was located at $0.1 c$ on both the pressure and the suction surfaces of the wing. This enabled the the present computational model to be designed such that laminar and turbulent boundary layer regions were replicated.

A multi-block, fully structured meshing technique was employed. Cells were concentrated near the boundaries and four chord lengths downstream of the trailing edge to ensure that the near-wake behavior was accurately represented. Cells were additionally concentrated near the endplate region to obtain the prominent upper and lower vortices. The $y^{+}$value remained below 1 over the wing, the endplate, and the ground plane. Three mesh densities were assessed at $h / c=0.179$ to determine the required resolution; an omnidirectional refinement ratio of 1.2 was applied to successive mesh densities. The medium mesh consisted of a total of $7.6 \times 10^{6}$ cells with 117 spanwise cells and 185 chordwise cells. The fine mesh and the coarse mesh consisted of $13.6 \times 10^{6}$ cells and $4.7 \times 10^{6}$ cells respectively. Examples of the mesh construction in an isometric view and at the symmetry plane are shown in Figure 3(a) and Figure 3(b) respectively. Efforts were particularly concentrated on ensuring that high-aspect-ratio cells existed only parallel to the flow at the boundary.

For all further cases (post-validation), the boundary layer was assumed to be fully turbulent, and the domain was extended in all directions. A boundary sensitivity study was undertaken and, as a result, the outlet was extended to $50 c$ downstream. The walls, the roof, and the inlet were also extended to a distance of $10 c$. Beyond these distances the aerodynamic forces ceased to change by more than $0.01 \%$. The mesh around the body was reflected about the $z$ axis and the $x$ axis to incorporate the whole geometry, as shown in Figure $3(\mathrm{a})$. This gave a total of $17.2 \times 10^{6}$ cells. The boundaries of the domain were modified to accommodate the path of the freestream flow, and the cells were also aligned in this direction. This transformation of the domain is shown in Figure 3(c) and (d). A rotating reference frame was used for all cases with flow curvature. The steady-state cornering condition was achieved by a constant angular velocity about a fixed point, external to the domain.

\section{Validation}

The results for the mesh study were generated using the $k-\omega$ shear stress transport (SST) turbulence model, ${ }^{24}$ coupled with a low-Reynolds-number boundary adaption. The different mesh sizes were found to have very little effect on prediction of the aerodynamic forces, shown in Table 1. From the medium mesh to the fine mesh, the aerodynamic forces did not change by more than $0.8 \%$. The higher concentration of cells across the span increased the resolution of the downstream wake, a noted benefit in the case of the medium mesh and the fine mesh.

The realizable $k-\varepsilon$ turbulence model $^{25}$ with the enhanced wall function was also assessed against the experimental data. The $k-\omega$ SST model was found to be particularly sensitive to the boundary layer mesh construction, and a slow and consistent growth rate away from the wall was required.

The results were assessed across nine ground clearances from $h / c=0.045$ to $h / c=0.448$, as shown in Figure 4. Particular emphasis was placed on whether 
Table I. Comparison of the experimental lift coefficients and the experimental drag coefficients for the coarse mesh, the medium mesh and the fine mesh at $h / c=0.179$.

\begin{tabular}{lll}
\hline Mesh & $C_{L}$ & $C_{D}$ \\
\hline Coarse & 1.228 & 0.052 \\
Medium & 1.241 & 0.052 \\
Fine & 1.248 & 0.052 \\
Experimental $^{12}$ & 1.28 & 0.055 \\
Medium (no transition) & 1.236 & 0.052 \\
\hline
\end{tabular}

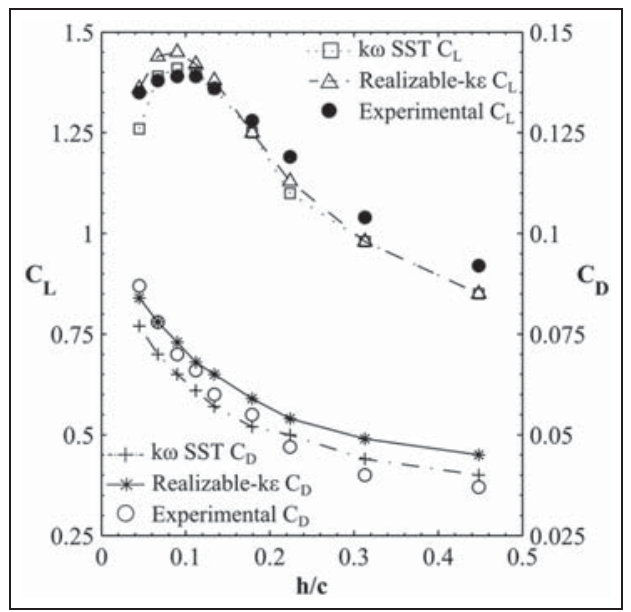

Figure 4. Drag coefficients and lift coefficients: comparison of the validation cases with the experimental results. SST: shear stress transport.

the turbulence models were capable of representing the trends in the aerodynamic forces from the published experimental results. At ground clearances above $h / c=0.224$, both models were found to under-predict lift and to over-predict drag. The over-prediction of drag was more severe in the case of the realizable $k-\varepsilon$ model, with the $k-\omega$ SST model matching the experimental results more closely. The under-prediction of lift was found to be largely attributed to an underprediction of the suction peak for both models at the higher ground clearances. Below $h / c=0.134$, both models predicted the early onset of vortex burst, associated with the 'downforce loss phenomenon'. ${ }^{10,13}$ In the case of the realizable $k-\varepsilon$, this separation was underpredicted, and the result was an over-prediction of the suction peak and lift in close proximity to the ground, before a severe loss in efficiency between $h / c=0.067$ and $h / c=0.045$. The $k-\omega$ SST model showed a similar behavior in close proximity to the ground but demonstrated a heightened level of sensitivity to separation induced by the adverse pressure gradient. As a result, lift predictions were matched more closely at low ground clearances despite the fact that the suction peak was also over-predicted.

An important point of difference for the two turbulence models is their abilities to match primary vortex
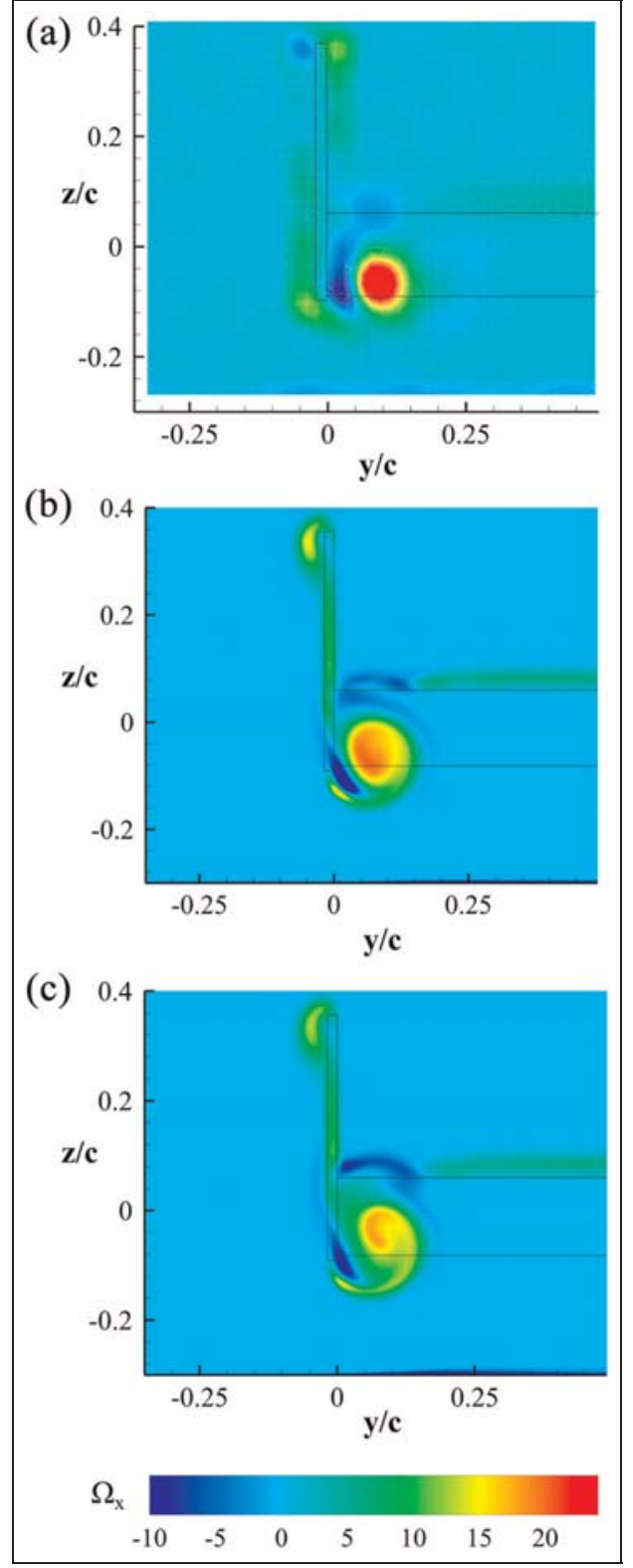

Figure 5. Streamwise vorticity contours for $h / c=0.224$ at $x / c=$ I.2: (a) experimental; ${ }^{12}$ (b) $k-\omega$ SST; (c) realizable $k-\varepsilon$.

formation. A comparison of streamwise vorticity contours at $x / c=1.2$, in which both models were compared with the published particle image velocimetry measurements, is shown in Figure 5. Both models were found to under-predict the maximum streamwise vorticity for the considered ground clearances. The strength of the counter-rotating vortical structures inside the endplate was also greater in the experiments and led to an increased distance between the endplate and the primary vortex, which is evident in Figure 5. Both numerical models predicted the development of the primary vortex to occur further downstream and this contributed toward general discrepancies. In the case of the realizable $k-\varepsilon$ turbulence model, this difference was more 
Table 2. Simulated cases in the present study.

\begin{tabular}{|c|c|c|c|c|c|}
\hline \multirow[t]{2}{*}{ Curvature } & \multicolumn{5}{|c|}{ Case designation for the following yaw angles } \\
\hline & $0^{\circ}$ & $1.25^{\circ}$ & $2.5^{\circ}$ & $5^{\circ}$ & $10^{\circ}$ \\
\hline 0 & $\mathrm{~S}$ & YI & Y2 & Y3 & Y4 \\
\hline $1 / 268.8 c$ & $\mathrm{Cl}$ & CRI & & & \\
\hline $1 / 134.3 c$ & $\mathrm{C} 2$ & & CR2 & & \\
\hline $1 / 67.2 c$ & C3 & & & CR3 & \\
\hline $1 / 33.6 c$ & C4 & & & & CR4 \\
\hline
\end{tabular}

significant. Both turbulence models matched the vortical structure; however, the $k-\omega$ SST model was clearly more closely correlated to the vorticity contours presented experimentally. With this structure forming a key point of investigation in the present work, the $k-\omega$ SST model was deemed to be favorable.

\section{Evaluation technique}

In reality the front wing is only one component of a complex system. In cornering-flow conditions the front wing will typically be in the yawed condition while also experiencing flow curvature. At the same time, other downstream components will observe different conditions.

The changes affected by cornering (the yaw and the curvature) were considered in isolation and then combined to give a realistic condition. This resulted in three different classes of cases, as shown in Table 2. As the cornering flow is variable throughout the domain, the described condition is that occurring at $x=0.5 c, y=0$ (halfway along the chord at the midspan location).

The Cartesian coordinate system was defined relative to wing itself rather than to the flow. This is because the other components on a car are positioned relative to the wing rather than to the flow. The velocity of the flow travelling in the $x$ direction at the wing's centre was $30 \mathrm{~m} / \mathrm{s}$ for all cases.

\section{Results}

\section{Near-wake structure}

The near-wake structure was highly sensitive to the yaw angle. As the yaw angle increased, the obstruction of the flow at the endplates increased. The oncoming flow began to observe more of the endplate side, rather than the edge, as shown in Figure 6. The effect was a change in the pressure distribution over the endplate surfaces, resulting in significant changes in the primary and secondary vortices, as well as in the vortical substructures.

The primary vortex remained below the suction surface, near the endplate for all conditions. The position of the centre of the primary vortex is shown for cases $\mathrm{S}$, C4, Y4, and CR4 in Figure 7.

For cases $\mathrm{Y}$ and $\mathrm{CR}$, the inboard vortex position was shifted toward the midspan location. The

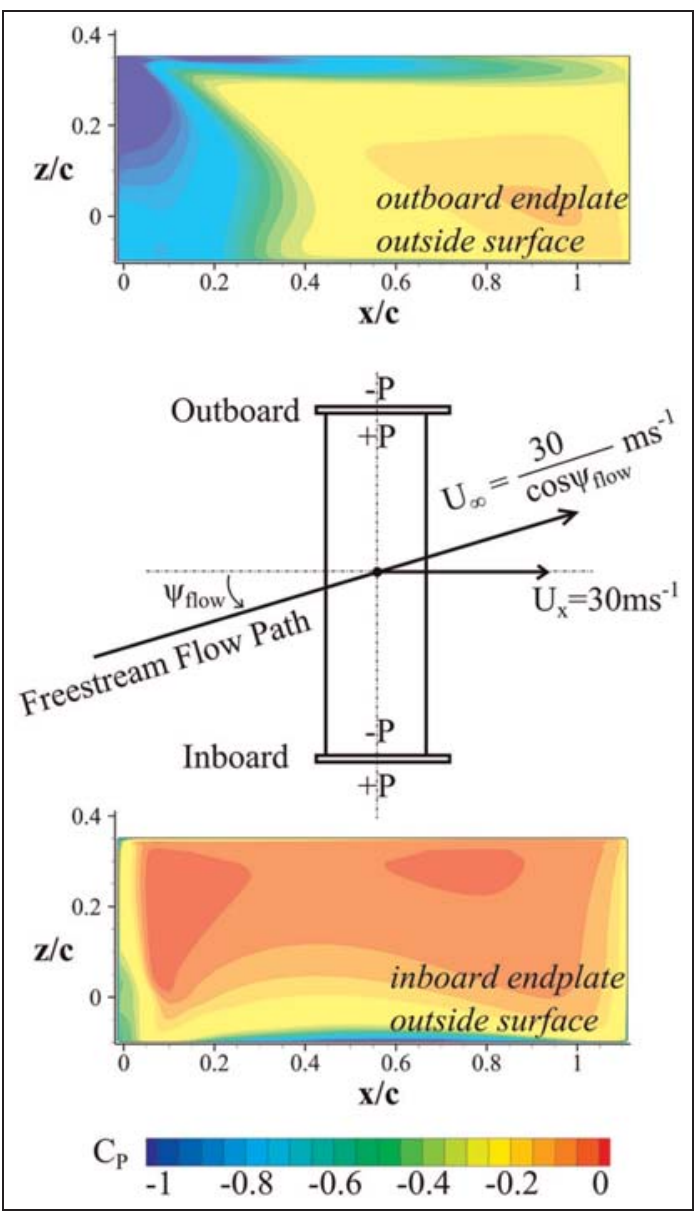

Figure 6. Change in the pressure occurring near the endplates owing to the flow angle and the pressure coefficient contours over the outside of the endplates for case Y4.

streamwise vorticity for the inboard primary vortex increased owing to the yaw angle, while a similar size was retained. This indicated an overall increase in the vortex strength. For cases Y4 and CR4, the peak streamwise vorticities increased by $66 \%$ and $43 \%$ respectively at $x / c=1.5$. The increase was asymptotic with the yaw angle. The induced shear from the ground and the endplate surfaces ultimately limited the size and the circulation.

The counter-rotating vortical substructure forming inside the inboard endplate became more prominent, as 


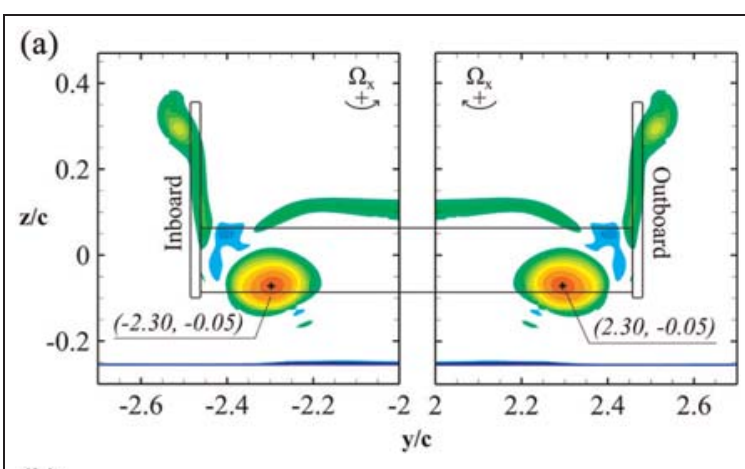

(b)

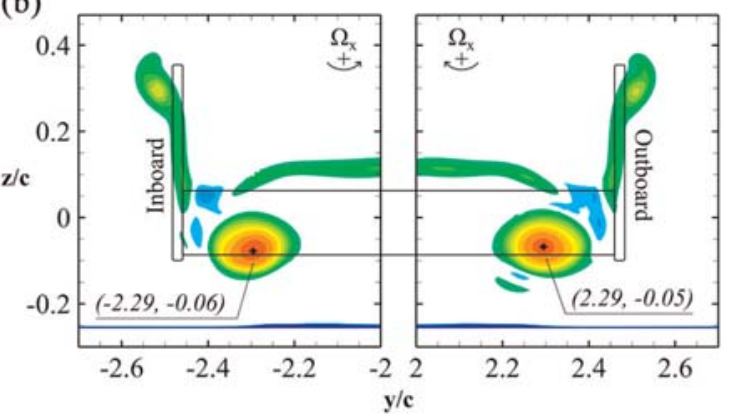

(c)

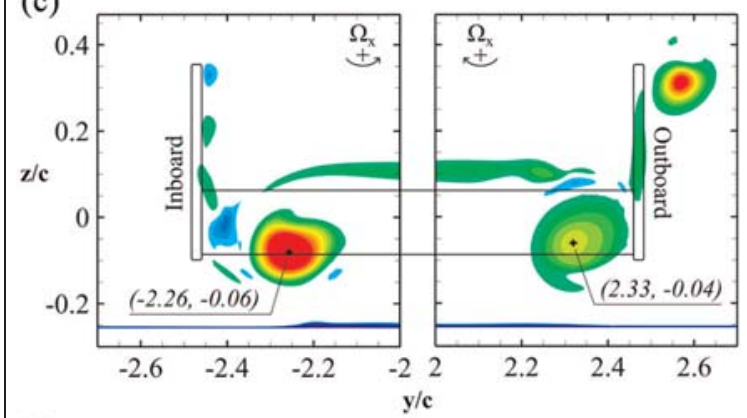

(d)

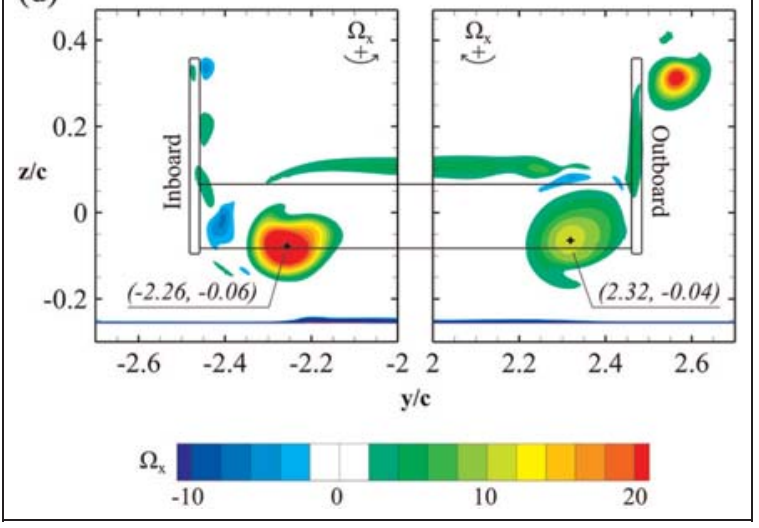

Figure 7. Streamwise vorticity at $x / c=1.5$ : (a) case S; (b) case C4; (c) case Y4; (d) case CR4.

shown in Figure 7(c) and (d). As the induced shear from the primary vortex increased, this smaller structure increased in strength. Similarly the counter-rotating vortex induced from the ground, as shown in Figure 8(a), increased in strength and was initiated earlier. In the straight-line condition, this was initiated from the

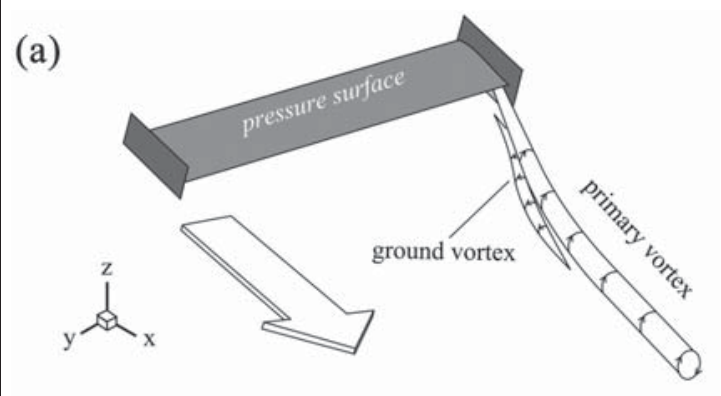

(b)

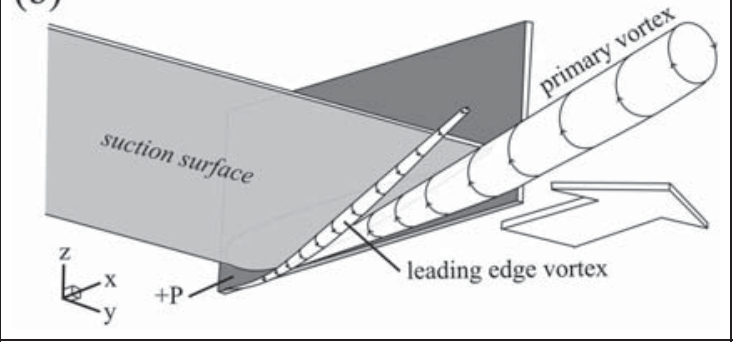

Figure 8. Positions of the prominent vortical substructures: (a) leading-edge vortex; (b) ground vortex.

ground at approximately $x / c=1.03$. At the maximum yaw angle the location was near $x / c=0.95$.

The strength of the outboard primary vortex decreased with increasing yaw angle. Decreases in the peak streamwise vorticities of $55 \%$ and $51 \%$ occurred for cases $\mathrm{Y} 4$ and CR4 respectively at $x / c=1.5$, as well as decreases in the size. The vortex remained in closer proximity to the endplates near the trailing edge. The relationship of the vorticity and the size was again asymptotic, decreasing as the yaw angle increased. The vortex was positioned higher than the inboard primary vortex for all yaw angles, and a notably smaller ground vortex formed near $x / c=2$.

In the straight-line case, the leading-edge vortex formed below the endplate and was drawn inside prior to the suction peak, as shown in Figure 8(b). As the yaw angle increased, the outboard leading-edge vortex became stronger owing to the increased pressure gradient. The location at which the vortex was drawn inside the endplate shifted downstream from $x / c=0.1$ to $x / c=0.45$. For cases $\mathrm{Y} 4$ and CR4, the leading-edge vortex passed below the already-formed primary vortex, affecting their interaction and causing the leadingedge vortex to circulate around the larger vortex. This contributed to the change in the primary vortex position relative to the endplate.

The leading-edge vortex also occurred inboard but was weaker and was positioned further inside the endplate. Consequentially, it interacted to a lesser extent with the primary vortex. In all cases the leading-edge vortex was inevitably exposed to a large axial adverse pressure gradient which led to breakdown prior to the trailing edge. Where the vortex was stronger, it was less susceptible and tended to continue further downstream. 


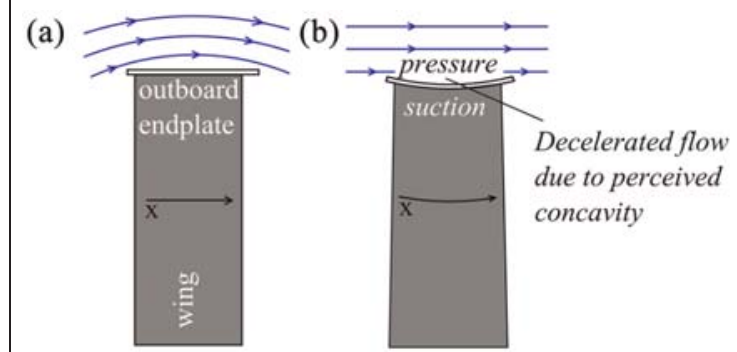

(c) 0.41

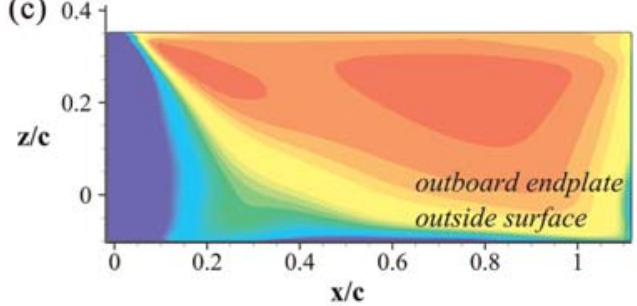

(d)

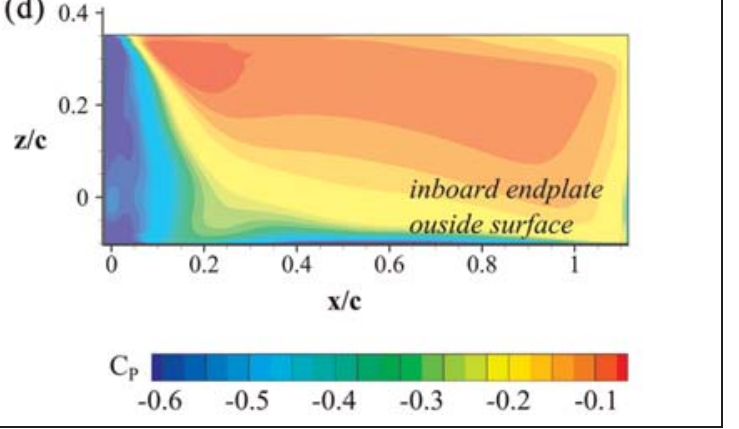

Figure 9. Effect of the perceived endplate curvature: (a) actual flow field conditions; (b) perceived curvature by flow (visualization purposes only); (c) pressure coefficient distribution over the outboard endplate outside surface for case C4;

(d) distribution over the inboard endplate outside surface for case C4.

The velocity gradient due to flow curvature also influenced the primary vortex strength. The freestream velocity increased with increasing distance from the centre of rotation, meaning that the outboard endplate was in a region of higher local $R e$, with the inboard lower. As a result the inboard primary vortex decreased in strength. The peak streamwise vorticity was $10 \%$ lower than in the straight-line condition. The opposite effect occurred for the outboard primary vortex. An increase in the vorticity occurred while the vortex retained the straight-line size and position. These velocity gradient effects explained why changes were less for cases CR than for cases $\mathrm{Y}$ at the same yaw angle.

The secondary vortices were more susceptible to changes in the freestream condition, as shown in Figure 7. As the yaw angle increased, the inboard secondary vortex was reduced in strength. This was due to the pressure gradient across the endplate. For cases Y3 and CR3 (a yaw angle of $5^{\circ}$ ), the inboard secondary vortex occurred in the form of two small counterrotating vortices which traveled above the endplate and merged near the trailing edge. The downstream path was inside the endplate, rotating with the opposite sign to the straight-line condition. For casesY4 and CR4, the vortex was clearly positioned inside the endplate and rotated in the opposite direction with the magnitudes of the peak vorticities being 35\% and 38\% higher than in the straight-line condition.

The outboard secondary vortex increased in strength as the summation of pressures had a net effect. The relationship was nearly linear with the yaw angle and also resulted in earlier development. Figure 7(c) and (d) clearly demonstrates that, for cases Y4 and CR4, the vortex is developed at $x / c=1.5$; this is not so for the same structure in the straight-line case and case $\mathrm{C} 4$.

The near wake was more affected by yaw than was the curved path of the flow in most instances. However, in cases $\mathrm{C}$, it was evident that the flow curvature affected how the geometry was perceived. Considering the relative motion of the flow past the endplate, as shown in Figure 9, the outside of the outboard endplate is observed as a concavity by the freestream flow. Effectively, the endplate had a pressure and a suction surface. This resulted in an increase in the pressure over the outside of the endplate and a decrease in the pressure inside.

For the outboard secondary vortex, this reduced the difference in the pressure across the upper half of the endplate. The peak streamwise vorticity of this vortex for case $\mathrm{C} 4$ decreased by $12 \%$ (at $x / c=1.5$ ), and the vortex was smaller, despite the fact that it was in a region of higher local $R e$. The inboard secondary vortex experienced the opposite effect. The flow curvature caused the endplate to be perceived to have the opposite curvature to that shown in Figure 9(a) and (b). This accelerated the flow and produced a lower pressure over the outside of the endplate, as shown in Figure 9(d). The peak streamwise vorticity was $10 \%$ higher, and a larger vortex indicated increased strength.

\section{Downstream vortex trajectories}

The downstream trajectory of vortices was affected by the freestream condition, as shown in Figure 10. The $Q$ criterion used in the figure is representative of the relationship between the rotation and the strain rate and has been shown to be an effective parameter for visualization of vortices. ${ }^{26}$ Measurement of the position in terms of the deflection in the $y$ direction highlighted a large change. The divergence of this path is of great importance and determines the position relative to the downstream components. In Figure 10, this divergence is measured relative to the freestream condition rather than to the Cartesian coordinate system, thus investigating the effect on the position independent of the freestream.

At both ends of the span, the endplates aligned the flow and vortices toward the $x$ direction, disrupting the freestream path. The vortex strength is related to the tendency of the structure to follow the direction of the 


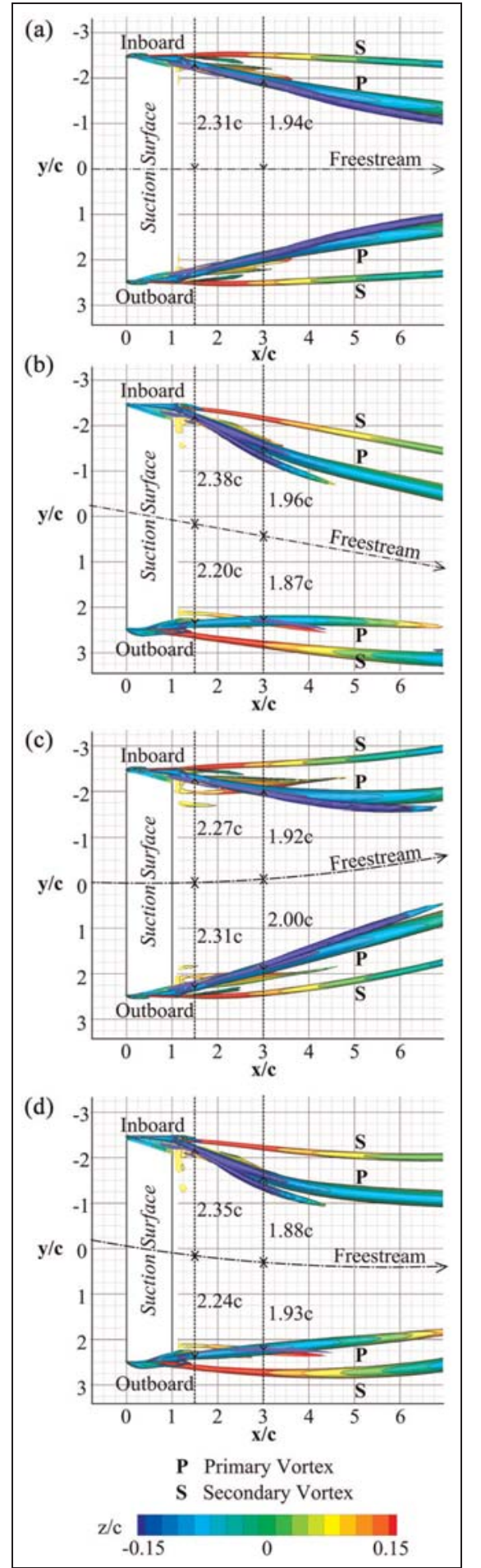

Figure 10. $Q$ criterion of 20000 , indicating the primary and secondary vortex paths which are colored according to the proximity of the ground: (a) case S; (b) case Y4; (c) case C4; (d) case CR4.

freestream flow. Figure 10(a) shows that, in the straight-line condition, the primary vortices follow a path approaching the midspan location while the secondary vortices aligned more closely with the endplates.
The increased strength of the primary vortex resulted in a path initially directed toward the ground and midspan locations. In Figure 10(b) and (d), this relationship is apparent, as the inboard primary vortex remained attached to the endplate but then angled across the span. The downward path of the inboard primary vortex for all cases $\mathrm{CR}$ and $\mathrm{Y}$ was ultimately limited as the vortex reached the ground. This also caused the crosssectional shape to become elliptical. The interaction with the ground boundary layer aligned the vortex back toward the freestream direction. The result was a more erratic curvature in its path.

The weaker outboard primary vortices retained or increased the ground clearance downstream for cases $\mathrm{CR}$ and $\mathrm{Y}$ and followed a path of slighter curvature. The outboard vortex was initially aligned with the endplate and then moved closer to the freestream centre, as shown in Figure 10(b) and (d). The trajectory retained a higher ground clearance than did the inboard vortex.

The inboard secondary vortices, in Figure 10(b) and (d), followed the freestream direction closely and retained a ground clearance similar to that of the straight line. Stronger outboard secondary vortices followed a wider arc and were directed downward. Cumulatively, these disparities also affected the position of the structures relative to each other.

For case C4 in Figure 10(c), and all cases C, the vortices followed a path similar to that of the straight line, albeit adjusted to the freestream condition. The vortices remained attached along the length of the endplate, meaning that the outboard vortices were positioned further away from the freestream centre. The flow curvature was greater inboard and, because of this, the endplate had a more significant straightening effect on the inboard vortex, with the deflection more significant than for the outboard vortex.

\section{Surface pressure and aerodynamic forces}

The distribution of the pressure over the wing surfaces was influenced by the local changes in $R e$. Figure 11 shows the pressure contours over the suction surface and the pressure plots at $y / c=-1.8$ and 1.8 .

The minimum pressure over the suction surface remained at the midspan location for all cases $\mathrm{Y}$, as shown in Figure 11(a). Both ends of the span had nearly identical magnitudes in the suction peak. Further aft, over the suction surface, there was an outboard increase in the pressure and an inboard decrease. This was largely attributed to the geometric shape of the endplate. In this instance the endplate extends only a small way below the wing surface at the location of the suction peak, reducing the interaction with the spanwise velocity component. Further aft, the endplate extends further below the suction surface, increasing the effect. The distribution was also affected on the pressure surface owing to the interaction of the flow with the endplates. 


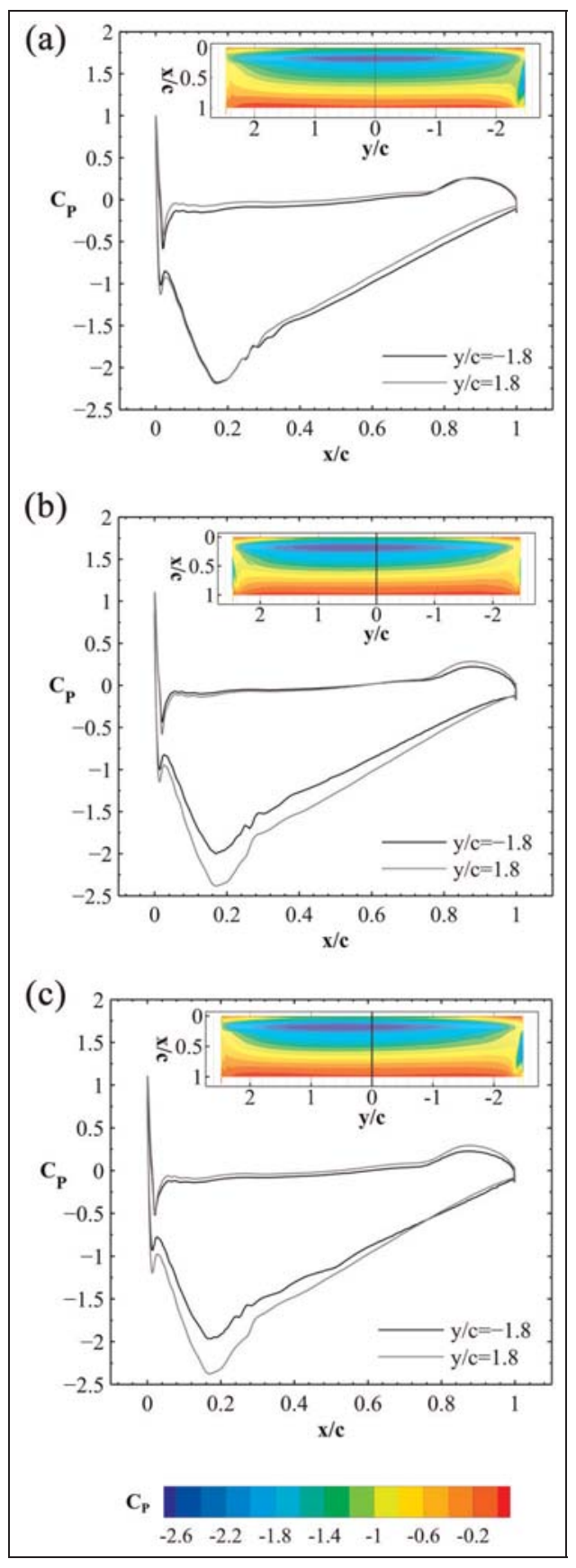

Figure II. Suction surface pressure coefficient and surface pressure plots: (a) case Y4; (b) case C4; (c) case CR4.

For cases $\mathrm{C}$, the outboard pressure distribution increased in magnitude owing to the local Re increase over this half of the span, as shown in Figure 11(b). From the contours on the suction surface, it can be seen that the minimum pressure over the suction surface clearly shifted outboard.

For cases CR, the pressure distribution was representative of the combined effects. The minimum pressure over the suction surface was shifted outboard and the suction peak increased.The imbalance in the pressure resulted in a net side force, rolling moment, and yawing moment about the aerodynamic centre. The side force, which is shown in Figure 12(a), increased linearly with increasing yaw angle. For cases Y4 and CR4, the side-force coefficient exceeded the straightline drag coefficient.

A negative rolling moment resulted for cases $\mathrm{C}$ and $\mathrm{CR}$ owing to the velocity gradient across the span. The curvature demonstrated a near-linear relationship with the magnitude of the moment for cases $\mathrm{C}$. The larger magnitude forces acting on the outboard half also increased production of the induced drag and resulted in a negative yawing moment.

The yaw angle resulted in a yawing moment which linearly increased with increasing flow angle, and a positive rolling moment which increased asymptotically. For cases CR, these forces were found to match very closely the sum of those observed for cases $\mathrm{C}$ and $\mathrm{Y}$. This demonstrated a level of independence between the effects of the flow angle and the velocity gradient.

In the present study, a reference velocity of $30 \mathrm{~m} / \mathrm{s}$ was established to ensure a consistent point of comparison, allowing simple calculation of the force based on the coefficients. Figure 12(d), (e) and (f) shows the lift and drag coefficient values for the three different case types. As a point of interest, the values were nondimensionalized in two different ways. The $C_{L}$ values were calculated using the constant reference velocity, whereas the corrected lift coefficient $C_{L C}$ accommodated the local change in $R e$ by employing the freestream variation.

For all cases, a difference of less than $1 \%$ existed in the negative lift force. This demonstrated a close relationship between the $x$ velocity component and the production of lift. It also highlighted that the vortexinduced effects were producing a net result of nearly zero. A stronger inboard vortex was always accompanied by a weaker outboard vortex, or vice versa. The corrected lift coefficient decreased with increasing yaw angle. For case CR4, the corrected lift coefficient was $4.7 \%$ lower than for the straight-line condition. The pitching moment of the wing remained within $0.6 \%$ for all cases with no clear trends.

As the yaw angle of the wing increased, the drag increased. This was predominantly due to the obstruction of the flow caused by the endplates. Cases Y4 and CR4 resulted in increases in the drag of $22.1 \%$ and $18.9 \%$ respectively. The magnitude of this difference increased exponentially with increasing yaw angle.

\section{Conclusion}

The steady-state cornering condition resulted in a fundamental change in the flow structures, which developed in the wake of an inverted wing in close proximity to the ground. This could feasibly result in a significant change in the aerodynamic performance of a vehicle when cornering. The most severe effects were attributed to the yaw angle which occurs during cornering; 


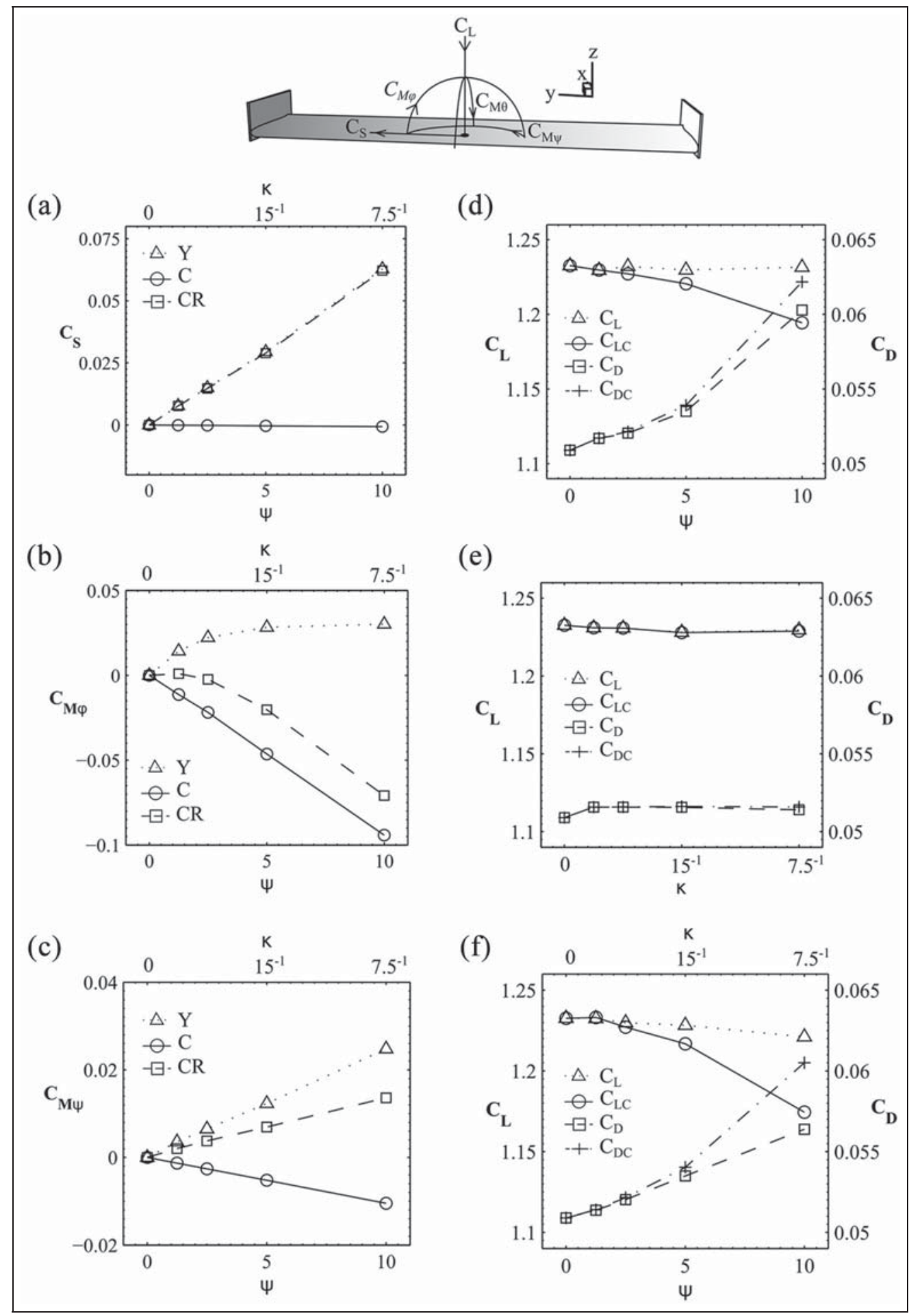

Figure 12. Aerodynamic force and moment coefficients acting on the wing: (a) side-force coefficients; (b) rolling moment coefficients; (c) yawing moment coefficients; (d) lift coefficients and drag coefficients for cases $Y$; (e) lift coefficients and drag coefficients for cases C; (f) lift coefficients and drag coefficients for cases CR.

however, both the flow curvature and the velocity gradient also affected the change.The difference in the pressure distributions near the endplates resulted in changes in the near-wake structure.
The wake became highly asymmetric with both primary and secondary longitudinal vortices differing in strength. The vortex core positions were also altered in both the vertical direction and the spanwise direction. 
These changes in position then became more substantial further downstream.

Smaller vortical substructures were observed to become more and less prominent according to the conditions. Their interaction with the primary vortex then affected the downstream trajectory of the larger structures.

An increase in the drag due to the spanwise flow component was the most significant change in the aerodynamic forces. Additionally, a yawing moment and a rolling moment occurred as an imbalance in the forces over the wing surface occurred owing to the local changes in $R e$.

In a practical situation, the wake of the front wing can have a significant effect toward the performances of the downstream components. The results highlight the potentially dramatic and cumulative effects that these sensitivities can have toward a vehicle's overall aerodynamic performance when cornering. Thus this demonstrates the importance of evaluating the cornering condition if that is where a good performance is most sought after.

\section{Declaration of conflict of interest}

The authors declare that there is no conflict of interest.

\section{Funding}

This research received no specific grant from any funding agency in the public, commercial, or not-for-profit sectors.

\section{References}

1. Zhang X, Toet W and Zerihan J. Ground effect aerodynamics of race cars. Appl Mech Rev 2006; 59: 33-49.

2. Katz J. Aerodynamics of race cars. A Rev Fluid Mech 2006; 38: 7-63.

3. Gordes A. Process for simulating curved airflow on wheeled vehicles in fluid channels with a straight measuring section. German Patent EP1610111A2, 2005.

4. Toet W. Aerodynamics and aerodynamic research in Formula 1. Aeronaut J 2013; 117(1187): 1-26.

5. Bradford J, Montomoli F and D'Ammaro A. Uncertainty quantification and race car aerodynamics. Proc IMechE Part D: J Automobile Engineering 2014; 228(4): 403-411.

6. Nara K, Tsubokura M, Ikeda J et al. A numerical analysis of unsteady aerodynamics of formula car during dynamic cornering motion. In: 32nd AIAA applied aerodynamics conference, Atlanta, Georgia, USA, 16-20 June 2014, AIAA paper 2014-3138. Reston, Virginia: AIAA.

7. Okada Y, Nouzawa T, Okamoto S et al. Unsteady vehicle aerodynamics during a dynamic steering action: 1st report, on-road analysis. SAE paper 2012-01-0446, 2012.

8. Tsubokura M, Ikawa Y, Nakashima T et al. Unsteady vehicle aerodynamics during a dynamic steering action: 2nd report, numerical analysis. SAE paper 2012-01-0448, 2012.
9. Agathangelou B and Gascoyne M. Aerodynamic design considerations of a Formula 1 racing car. SAE paper 980399, 1998

10. Zhang $X$ and Zerihan J. Off-surface aerodynamic measurements of a wing in ground effect. J Aircr 2003; 40(4): 716-725.

11. Dominy RG. Aerodynamics of Grand Prix car. Proc IMechE Part D: J Automobile Engineering 1992; 206(4): 267-274.

12. Zerihan J. An investigation into the aerodynamics of wings in ground effect. PhD Dissertation, University of Southampton, Southampton, UK, 2001.

13. Zerihan $\mathbf{J}$ and Zhang $\mathbf{X}$. Aerodynamics of a single element wing in ground effect. J Aircr 2000; 37(6): 10581064.

14. Zhang $X$ and Zerihan J. Aerodynamics of a double element wing in ground effect. AIAA $J$ 2003; 41(6): 1007-1016.

15. Diasinos S, Barber TJ and Doig G. Influence of wing span on the aerodynamics of wings in ground effect. Proc IMechE Part G: J Aerospace Engineering 2013; 227(3): 569-573.

16. Soso $\mathrm{M}$ and Wilson P. Aerodynamics of a wing in ground effect in generic racing car wake flows. Proc IMechE Part D: J Automobile Engineering 2006; 220(1): 1-13.

17. ANSYS $^{\circledR}$ Fluent, release 14.5.Canonsburg, Pennsylvania: ANSYS, Inc.

18. Mahon SA and Zhang X. Computational analysis of pressure and wake characterisitcs of an aerofoil in ground effect. Trans ASME, J Fluids Engng 2005; 127: 290-298.

19. Doig G, Barber TJ and Neely AJ. The influence of compressibility on the aerodynamics of an inverted wing in ground effect. Trans ASME, J Fluids Engng 2011; 133(6): $1-12$.

20. Doig $\mathrm{G}$ and Barber T. Considerations for numerical modelling of inverted wings in ground effect. AIAA $J$ 2011; 49(10): 2330-2333.

21. Doig G, Barber TJ, Leonardi E and Neely AJ. The onset of compressibility effects for an inverted aerofoil in ground effect. Aeronaut $J$ 2007; 111(1126): 797-806.

22. Keogh J, Diasinos S and Doig G. Flow compressibility effects around an open-wheel racing car. Aeronaut $J$ 2014; 118 (1209): 1-23.

23. Barber TJ, Leonardi E and Archer RD. Causes for discrepancies in ground effect analyses. Aeronaut $J$ 2002; 106(1066): 653-657.

24. Menter FR. Two-equation eddy-viscosity turbulence models for engineering applications. AIAA J 1994; 32: 269-289.

25. Shih TH, Liou WW, Shabbir A et al. A new $k-\varepsilon$ eddyviscosity model for high Reynolds number turbulent flows - model development and validation. Comput Fluids 1995; 24(3): 227-238.

26. Hunt JCR, Wray AA and Moin P. Eddies, streams, and convergence zones in turbulent flows. In: Studying turbulence using numerical simulation databases, proceedings of the 1988 summer program, Vol 2(1). Report CTR-S88, Centre for Turbulence Research, Stanford University, Staford, California, USA, 1988, pp. 193-208. 


\section{Appendix I}

\section{Notation}

b $\quad$ span $=1100 \mathrm{~mm}$

$c \quad$ chord length $=223.4 \mathrm{~mm}$

$C_{D} \quad$ coefficient of force in the direction aligned with the freestream

$C_{D C}$

$C_{L}$

$C_{L C}$

$C_{P}$

$C_{M \theta}$

$C_{M \varphi}$

$C_{M \psi}$

$C_{S}$

$Q$

$U_{\infty}$

$x, y, z$ coefficient of drag, corrected according to local the freestream velocity magnitude coefficient of force in the negative $z$ direction

coefficient of lift, corrected according to the local freestream velocity magnitude coefficient of the pressure coefficient of the pitching moment coefficient of the rolling moment coefficient of the yawing moment coefficient of force in the positive $y$ direction

$Q$ criterion, second invariant of the velocity grade tensor $\nabla u$

freestream velocity $(\mathrm{m} / \mathrm{s})$

Cartesian right-handed coordinates

turbulent dissipation

angle of incidence about the $y$ axis flow curvature $\left(\mathrm{m}^{-1}\right)$

angle of incidence about the $x$ axis angle of incidence about the $z$ axis specific dissipation rate non-dimensional vorticity about the $x$ axis 